\title{
Fragilizzare, fragilizzante e fragilizzazione
}

\author{
Giuseppe Sergio
}

PUBBLICATO: 16 APRILE 2021

\section{Quesito:}

Alcuni lettori ci segnalano le forme anti-fragilizzante e fragilizzati, detto dei capelli, usate in alcune pubblicità di prodotti cosmetici: sono corrette? Un altro lettore ci chiede se la parola fragilizzazione sia utilizzabile anche al di fuori di ambiti tecnico-scientifici.

\section{Fragilizzare, fragilizzante e fragilizzazione}

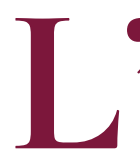

aggettivo e participio passato fragilizzato è formato a partire dalla base aggettivale fragile, cui si applica il suffisso -izzato. Questo tipo di derivazione è oggi molto produttivo sia nella lingua comune (per es. opaco $\rightarrow$ opacizzato), sia, soprattutto, in ambiti tecnico-settoriali, cui sono da ricondursi la maggior parte degli impieghi (per es. digitale $\rightarrow$ digitalizzato, lessicale $\rightarrow$ lessicalizzato). Il tipo è inoltre diffuso nella lingua dei media, in particolare del giornalismo e della pubblicità, dove viene gradito per l'alone tecnicistico del suffisso -izzato e per la sua sinteticità, che consente di aggirare perifrasi (nel caso in specie, capelli resi o diventati fragili $\rightarrow$ capelli fragilizzati). Insieme ai corradicali fragilizzare, fragilizzante e fragilizzazione, fragilizzato costituisce una famiglia lessicale coesa sia dal punto di vista formale sia da quello semantico; come accade per i verbi deaggettivali, essa è accomunata dal passaggio dal non possedere una proprietà (quella indicata dall'aggettivo) all'averla: nel nostro caso, dal non essere fragile all'esserlo.

\section{Storia}

La voce fragilizzato non è accolta nei più importanti dizionari dell'uso, né compare in quelli storici; viene invece registrata fra i Neologismi del Vocabolario Treccani (2008; ancor prima nei Neologismi quotidiani, Firenze 2003) e nel repertorio dell'ONLI - Osservatorio Neologico della Lingua Italiana, che indicano come significato 'reso fragile'. Gli esempi riportati da queste due fonti - tratti dalla stampa periodica, risalenti agli anni 2000 (Treccani) e al decennio successivo (ONLI) - fanno riferimento a contesti economici, politici, medico-psicologici e anche senzaltro comuni, come nel seguente esempio: "La posizione personale di Lionel Jospin è fragilizzata anche a causa di una vicenda molto ambigua" (da "Il Fatto", 2002). Mentre Treccani avverte che la voce si trovava già, sulla "Repubblica", nel I987, ONLI retrodata la prima attestazione al I95I, quando compare sulla "Stampa".

Attentandoci nel pur infido terreno di Google libri, la prima attestazione di fragilizzato può essere retrodata addirittura a inizio Novecento (precisamente al I9I3, con attestazione sullo "Sperimentale: archivio di biologia normale e patologica"), trovando nel corso del secolo ulteriori attestazioni, oltre che in ambito medico, anche in quello chimico e soprattutto metallurgico. Il pedigree medico e l'attestazione ai primi decenni del Novecento appaiono confermati, sempre stando a Google libri, anche dalle ricerche delle forme fragilizzata (I936, su "Le Monde Médical. Rivista internazionale di medicina e terapia"), fragilizzati (1913, sullo "Sperimentale: archivio di biologia normale e patologica"), fragilizzate (I9I2, sulla "Gazzetta degli ospedali e delle cliniche"), fragilizzante (I924, nella Patologia e chirurgia della milza di Luigi Silvestrini) e fragilizzazione (I915, sulla "Clinica medica italiana").

La prima attestazione, o una delle prime, in letteratura si troverebbe invece in Una sensibilitá italiana 
nata in Egitto, memoriale postumo di Filippo Tommaso Marinetti, in buona parte dettato fra il I943 e il I944:

Il meriggio settembrino [...] appesantiva nell'aria strambi calvari di smisurate croci di torrido ottone massiccio ed erano spessori di raggi in forma di croci tali da frantumare le nostre mani fragilizzate o scavare le nostre spalle di poveri Cristi umani. (F. T. Marinetti, La grande Milano tradizionale e futurista. Una sensibilitá italiana nata in Egitto, Milano, Mondadori, 1969, pp. 219-220)

Le voci fragilizzato ecc. non risultano dunque neologiche in senso assoluto, essendo attestate in italiano almeno dai primi decenni del secolo scorso, seppur in prevalenza rifugiate in ambiti tecnicoscientifici, in particolare medico, chimico e metallurgico.

\section{Diffusione attuale}

In anni a noi più recenti fragilizzato sembra aver perso la sua valenza più specialistica, tracimando in ambiti più genericamente settoriali, quando non senz'altro comuni. Ciò si deve alla sua trasparenza semantica e al traino della rinnovata circolazione di fragile, diffusosi come sostituto eufemistico di 'problematico', 'a rischio' ecc.: non sono infrequenti, infatti, i riferimenti a categorie e a soggetti fragili o con fragilità, a zone della fragilità sociale ecc.; si pensi anche, in tempi di Covid, alla diffusione del sintagma lavoratori fragili, riferito a lavoratori non più giovani o con patologie pregresse.

Una ricerca delle quattro forme participiali fragilizzato/-a/-i/-e nella banca dati Factiva dell'editore Dow Jones - che relativamente alla lingua italiana copre le maggiori testate giornalistiche, nazionali e locali, dal 1996 a oggi - ha mostrato una certa vitalità della voce nella stampa più recente, dove ricorre, a partire dallo stesso 1996 fino ad oggi, IO4 volte. Gli ambiti d'uso in cui è prevalentemente allignata sono quello politico e quello economico: dal primo si contano 36 contesti (per es. "Macron e Merkel [...] sono obbligati a difendere l'asse franco-tedesco, seriamente fragilizzato", "Il Messaggero", 29/5/2019), dal secondo 35 (per es. "Basta spostarsi di poco dal centro della capitale libanese, di giorno, per vedere gli effetti del blocco causato dalla pandemia su un'economia già fragilizzata dalla crisi del debito pubblico", "Euronews", 4/6/2020). L'ambito politico e quello economico coprono, insieme, oltre i due terzi dei contesti totali; più limitati sono le occorrenze in altri settori: quello medicopatologico, soprattutto in relazione a problemi psicologici (per es. "a volte in persone fragilizzate dalla grave depressione [...] la determinazione al suicidio scatta per le situazione di anonimato e di solitudine percepita", ANSA, 4/2/20I7), quello ecologico-ambientale ("Prati e campi devastati, cotica erbosa divelta, profondi scavi che rendono pericolanti i muretti di contenimento del terreno in un territorio già di per sé fragile e fragilizzato da decenni di abbandono", "La Sentinella del Canavese", I7/8/2020), quello sociale ("Di fronte alle nostre comunità che si sono cosi fortemente fragilizzate [...] non è pensabile tentennare ancora", ANSA, 8/4/2020) e quello cosmetico ("E proprio al concetto di delicatezza di low poo si è ispirata L'Oréal Paris per il nuovo rituale di bellezza Elvive specifico per capelli colorati, secchi e fragilizzati", "la Repubblica", I9/8/I7). Seppur limitatamente, la diffrazione del termine si è spinta fino ad alcuni contesti da ritenersi comuni, come nel seguente esempio che compare, con significativa virgolettatura, all'interno di un'intervista: «Non mi dava punti di riferimento, mi ha "fragilizzata"» ("Corriere della Sera", I3/9/2015).

\section{Pubblicità e commercio}

Gli impieghi commerciali e pubblicitari di fragilizzato si ascrivono alla cura dei capelli e della pelle. A quest'ultimo ambito rimandano per esempio le uniche due occorrenze rintracciate nell'archivio digitale di "Vogue Italia", in entrambi i casi riferite a creme per il viso: 
Comfort Extreme Jour evita alle pelli secche o temporaneamente fragilizzate la sgradevole sensazione di tensione e disagio (pubblicità Sisley, marzo 2004);

Skin Life Repair, super nutriente per pelli aride e fragilizzate dall'età, Helena Rubinstein (didascalia, febbraio 2007).

Concentrandoci sui prodotti per i capelli, da cui prendono spunto le richieste dei lettori, in commercio si trovano linee che fin dall'etichetta sono raccomandate per "capelli secchi, fragilizzati" (Elvive Olio Straordinario Low Shampoo), per "capelli colorati, fragilizzati" (Color Elvive Low Shampoo; dove si noterà anche il neologismo Low shampoo 'shampoo delicato') oppure più semplicemente "per capelli fragili" (Botanicals Fresh Care; tutte e tre le linee citate sono prodotte da L'Oréal).

A spiegare l'utilizzo di fragilizzato si possono chiamare in causa diversi fattori, legati alla psicologia della vendita: fra questi il richiamo della suffissazione in -izzato, che avvalora la credibilità del prodotto suggerendone l'efficacia tecnicistica; la deresponsabilizzazione del consumatore attraverso una sorta di "fattore lusinga", in base al quale la causa della fragilità non sarebbe intrinseca al capello, e dunque al suo possessore, ma derivata da agenti esterni (detto altrimenti, i capelli non sono senz'altro fragili, ma resi tali da trattamenti, colorazioni, agenti atmosferici, sole e salsedine ecc.); il baluginio derivato dalla novità formale del termine, che per cosi dire rivernicia a nuovo un referente noto (fragilizzati, infatti, non sono altro che i capelli che altrimenti si dicono indeboliti, danneggiati o sfibrati).

La novità formale, in particolare, crea una differenza percettiva sia rispetto ai cosiddetti competitors, cioè i prodotti simili che si fanno diretta concorrenza, sia rispetto a prodotti che il consumatore può aver acquistato in passato. La scelta di uno shampoo per capelli fragilizzati può dunque avvenire dopo aver provato, magari senza grandi risultati, shampoo per capelli danneggiati (cosi per esempio sulle etichette di altri shampoo delle linee dedicate di Elvive, Sunsilk, Pantene, Gliss), stressati ("capelli stressati, inclini alle doppie punte", Gliss linea Supremo Oil Elixir; "capelli stressati dall'estate", Gliss linea Summer Repair) o sfibrati (Arkalia, Restivoil).

Considerato l'elevato internazionalismo che contraddistingue gli ambiti commerciali e pubblicitari, alla diffusione di fragilizzato potrà aver concorso anche l'influsso di modelli stranieri. Difficile stabilire con certezza se l'input provenga dall'inglese o dal francese, anche se può ipotizzarsi una più probabile derivazione francese. Oltre a considerazioni extralinguistiche, legate alla tradizionale supremazia della Francia nella cosmetica, supremazia peraltro sempre più intaccata dall'eccellenza laboratoriale angloamericana, farebbero propendere per un influsso francese ragioni più propriamente storicolinguistiche. Infatti, mentre to fragilize e la forma participiale fragilized apparirebbero di più recente corso, tanto da non essere ancora accolti nell'Oxford English Dictionary OED, in francese si registrano non solo fragiliser (cfr. TLFi, con prima attestazione al 1956 ed esempi di ambito medico-patologico), ma anche l'aggettivo fragilisant e il sostantivo fragilisation (attestati da TLFI rispettivamente al ig67 e al I965, sempre in ambito medico).

Una riprova di un più probabile influsso francese si ricaverebbe dal confronto fra quanto si legge sulle confezioni di alcune linee di prodotti per capelli commercializzate da L'Oréal sui mercati italiano, francese e inglese. Se per la linea Elvive Full Resist la versione italiana si allinea a quella inglese (lo shampoo è indicato, rispettivamente, per "capelli fragili, tendenti a caduta" e per "fragile hair, tendency to break and fall"), mentre quella francese opta per "cheveux fragilisés, tendance à tomber", per altre linee l'italiano segue il francese fragilisé impiegando fragilizzato. Questo accade per Elvive Olio Straordinario Low Shampoo, indicato per "capelli secchi, fragilizzati", che ricalca, della stessa 
linea, il francese "cheveux secs | fragilisés" (di contro all'inglese "dry, fragile hair") e per Color Elvive Low Shampoo per "capelli colorati, fragilizzati", più aderente alla dizione francese "cheveux colorés | fragilisés", mentre l'inglese presenta "dry, coloured hair".

\section{Conclusioni}

In definitiva non paiono esserci dubbi sull'accettabilità difragilizzato, così come difragilizzare, fragilizzante efragilizzazione. Si tratta di voci ben formate e semanticamente trasparenti che si inseriscono in una classe, quella dei deaggettivali in -izzare, in espansione nell'italiano contemporaneo. L'unico difetto, se tale può considerarsi, risiede nella loro possibile cacofonia, che però non appare intrinseca, bensi collegata alla loro circolazione ancora limitata, seppur in espansione d'uso. D'altro canto può infatti prevedersi che, anche e proprio grazie allimpiego in ambito cosmetico, amplificato dalla pubblicità e dal commercio, le voci possano presto conoscere una diffusione a più ampio raggio.

\section{Cita come:}

Giuseppe Sergio, Fragilizzare, fragilizzante e fragilizzazione, "Italiano digitale", XVII, 2021/2 (aprilegiugno)

DOI: $10.35948 / 2532-9006 / 2021.6518$

Copyright 2021 Accademia della Crusca

Pubblicato con licenza creative commons CC BY-NC-ND 